\title{
The Earth's passage of the April 11, 1997 coronal ejecta: geomagnetic field fluctuations at high and low latitude during northward interplanetary magnetic field conditions
}

\author{
S. Lepidi ${ }^{1,2}$, P. Francia ${ }^{1}$, U. Villante ${ }^{1}$, A. Meloni ${ }^{2}$, A. J. Lazarus ${ }^{3}$, R. P. Lepping ${ }^{4}$ \\ ${ }^{1}$ Dipartimento di Fisica, Università dell'Aquila, L'Aquila, Italy \\ E-mail: stefania.lepidi@aquila.infn.it \\ ${ }^{2}$ Istituto Nazionale di Geofisica, Roma, Italy \\ ${ }^{3}$ Center for Space Research, MIT, Cambridge, USA \\ ${ }^{4}$ Laboratory for Extraterrestrial Physics, NASA/GSFC, Greenbelt, USA
}

Received: 18 December 1998 / Revised: 21 April 1999 / Accepted: 10 May 1999

\begin{abstract}
An analysis of the low frequency geomagnetic field fluctuations at an Antarctic (Terra Nova Bay) and a low latitude (L'Aquila, Italy) station during the Earth's passage of a coronal ejecta on April 11, 1997 shows that major solar wind pressure variations were followed at both stations by a high fluctuation level. During northward interplanetary magnetic field conditions and when Terra Nova Bay is close to the local geomagnetic noon, coherent fluctuations, at the same frequency $(3.6 \mathrm{mHz})$ and with polarization characteristics indicating an antisunward propagation, were observed simultaneously at the two stations. An analysis of simultaneous measurements from geosynchronous satellites shows evidence for pulsations at approximately the same frequencies also in the magnetospheric field. The observed waves might then be interpreted as oscillation modes, triggered by an external stimulation, extending to a major portion of the Earth's magnetosphere.
\end{abstract}

Key words. Magnetospheric physics (MHD waves and instabilities; solar wind-magnetosphere interactions)

\section{Introduction}

Interplanetary magnetic clouds, which are an important subset of coronal mass ejections (Gosling, 1990), are typically characterized by strong magnetic field intensities and smooth rotation of the magnetic field direction through large angles over distances of $0.25-1$ AU (Burlaga, 1991). Several investigations have recently analyzed the interaction between coronal ejecta, characterized by long periods of out-of-ecliptic interplane-

Correspondence to: $\mathrm{S}$. Lepidi tary magnetic field (IMF) orientation, and the Earth's magnetosphere. While the prolonged southward IMF orientations have been confirmed to be associated with major geomagnetic storms (Lepping et al., 1991; Farrugia et al., 1993, 1996), during northward IMF conditions (i.e. during closed magnetospheric conditions, when transient effects such as the onset of substorm current systems and the intensification of the ring current can be neglected) it clearly emerges that there is a correspondence between solar wind (SW) density enhancements, magnetospheric compressions and variations of the ground magnetic field (Francia et al., 1999; see also Sibeck et al., 1989; Russell et al., 1994; Russell and Ginskey, 1995).

In a recent paper (Villante et al., 1998) we analyzed the geomagnetic field variations at a low latitude (L'Aquila, Italy, AQ) and an Antarctic station (Terra Nova Bay, TNB) associated with the Earth's passage of the January 10-11, 1997 magnetic cloud. At both sites we found, in addition to sudden impulses and storm signatures, a high level of fluctuations following the major SW pressure variations; in particular, during closed magnetospheric conditions, low frequency oscillation modes at the same discrete frequencies were observed at the two stations, suggesting that strong SW pressure pulses can also generate global oscillations of the whole magnetosphere (Walker et al., 1992). Evidence for such long-period fluctuations has already been proposed in previous statistical studies of ULF oscillations at TNB (Villante et al., 1997) and AQ (Francia and Villante, 1997).

Here we conduct a study of the geomagnetic field fluctuations observed at $\mathrm{AQ}$ and TNB during the passage of a solar ejecta (April 11, 1997), characterized by northward IMF conditions and strong pressure variations. Our results show at the two stations simultaneous power intensifications at low frequencies, triggered by SW pressure pulses. In addition, oscillations with the same discrete frequencies and high coherence between the two stations can be observed during closed magnetospheric conditions when TNB is close to the 
local geomagnetic noon and AQ is in the evening sector; this result can be interpreted in terms of compressional oscillations which extend to a major portion of the Earth's magnetosphere.

\section{Experimental observations}

In this study we analyzed the 1 min averages of the horizontal magnetic field components $\mathrm{H}$ and $\mathrm{D}$ measured at AQ (IGRF95 corrected geomagnetic coordinates: $\left.36.2^{\circ} \mathrm{N}, 87.5^{\circ} \mathrm{E} ; \mathrm{MLT}=\mathrm{UT}+1: 40\right)$ and $\mathrm{TNB}$ $\left(80.0^{\circ} \mathrm{S}, 307.7^{\circ} \mathrm{E}\right.$; MLT $\left.=\mathrm{UT}-8: 06\right)$. Both instruments consist of three orthogonal fluxgate magnetometer sensors sampled respectively at $1 \mathrm{~s}$ (AQ) and $30 \mathrm{~s}$ (TNB) with a flat frequency response in the range $0-1$
Hz. For the SW plasma and IMF data we used the key parameters from WIND spacecraft.

In Fig. 1 we show the $\mathrm{H}$ and $\mathrm{D}$ components at $\mathrm{AQ}$ and TNB and the simultaneous interplanetary data recorded on April 11, 1997. As discussed by Berdichevsky et al. (1998), a solar ejecta was detected from WIND at $0550 \mathrm{UT}$, at a geocentric distance of $\approx 230 \mathrm{Re}$ in the Earth-Sun direction. It is preceded, between $\approx 0440$ and 0550 UT, by a compression region with plasma densities up to $\approx 35 \mathrm{~cm}^{-3}$. The ejecta is well identified by a sharp variation of the plasma and IMF parameters; in particular, the IMF strength increases from $\approx 8 \mathrm{nT}$ to $\approx 20 \mathrm{nT}$ and its direction becomes definitely northward: $\Theta$ increases from $\approx-45^{\circ}$ to more than $65^{\circ}$ and then, except for a rapid southward switch before $\approx 1800 \mathrm{UT}$, it remains positive until $\approx 1930$ UT. Within the ejecta, the

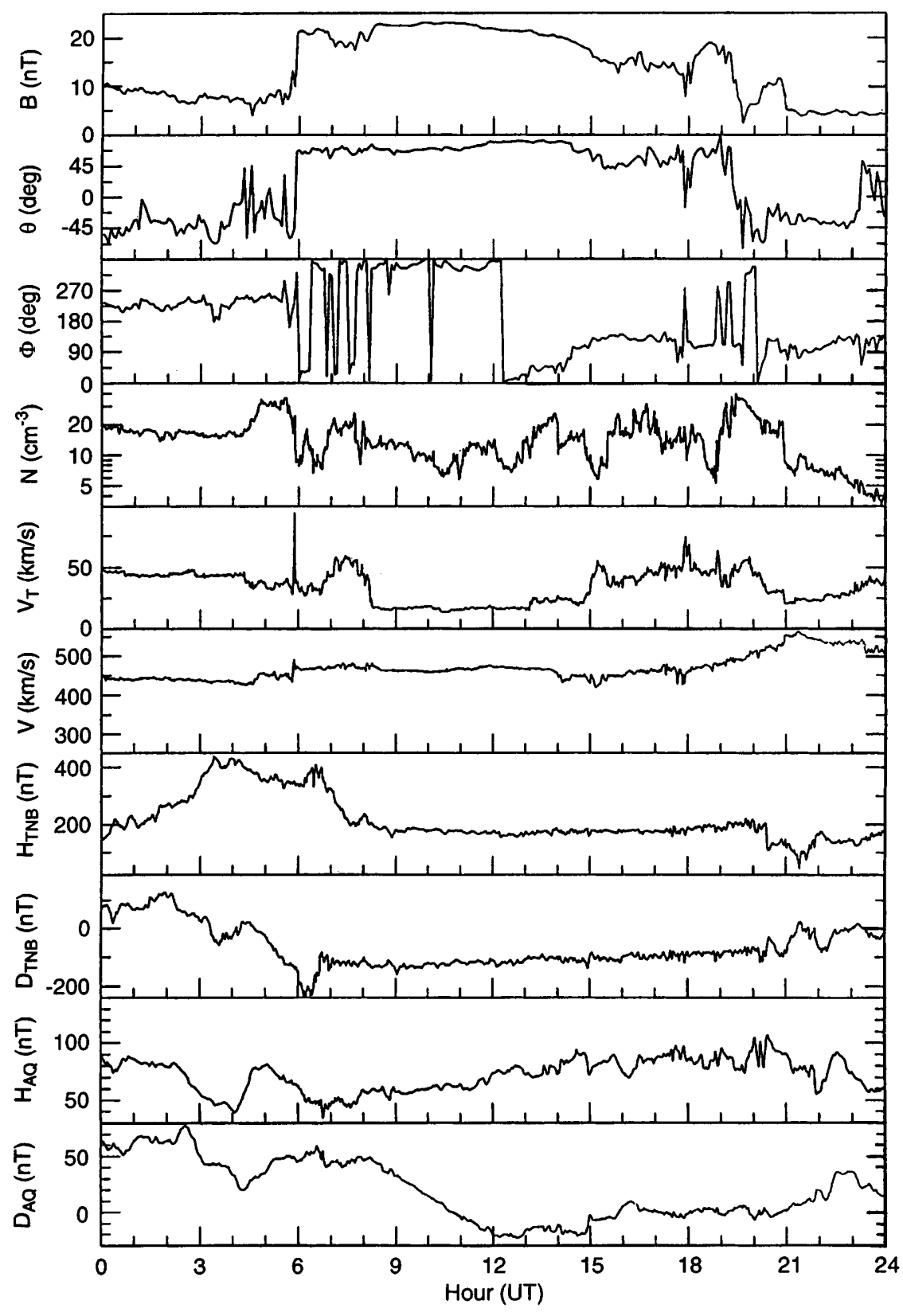

Fig. 1. WIND and ground observations on April 11, 1997. From top to bottom are shown the magnetic field strength and its latitude and longitude, the proton number density and thermal speed, the SW flow speed and the geomagnetic field components $\mathrm{H}$ and $\mathrm{D}$ at TNB and AQ 
SW speed is quite steady, with values of $\approx 470 \mathrm{~km} / \mathrm{s}$, while the plasma density shows several variations. Following the ejecta, after $\approx 1500 \mathrm{UT}$, there is a region characterized by enhanced and variable field intensity and pressure; in particular, strong and sharp increases of the SW density are detected at $\approx 1530$ (from $\approx 8 \mathrm{~cm}^{-3}$ to $\approx 17 \mathrm{~cm}^{-3}$ ) and $\approx 1900$ UT (with peak values of $\approx 35 \mathrm{~cm}^{-3}$ ); this is evidently an interaction region formed ahead of the high speed stream present late on April 11 (Berdichevsky et al., 1998).

The Earth's arrival of the ejecta, with the northward turning of the IMF (the propagation time from WIND to the Earth is of the order of $50 \mathrm{~min}$ ), can be identified at TNB at $\approx 0640$ UT by a sharp geomagnetic field variation (less clear at AQ), which is more evident on the $\mathrm{D}$ component. In the following hours, when the IMF remains constantly northward, the geomagnetic field level at TNB is quite stable, while at AQ it shows the usual daily variation (Meloni and Molina, 1989). At both stations, a superimposed high level of fluctuation activity is evident in this time interval. The southward turning of the IMF after the ejecta $(\approx 1930$ UT) seems to be accompanied by a sharp negative variation of the $\mathrm{H}$ component at TNB $(\approx 2024$ UT).

We performed an analysis of the low frequency fluctuation power for the whole day. In Fig. 2a we show
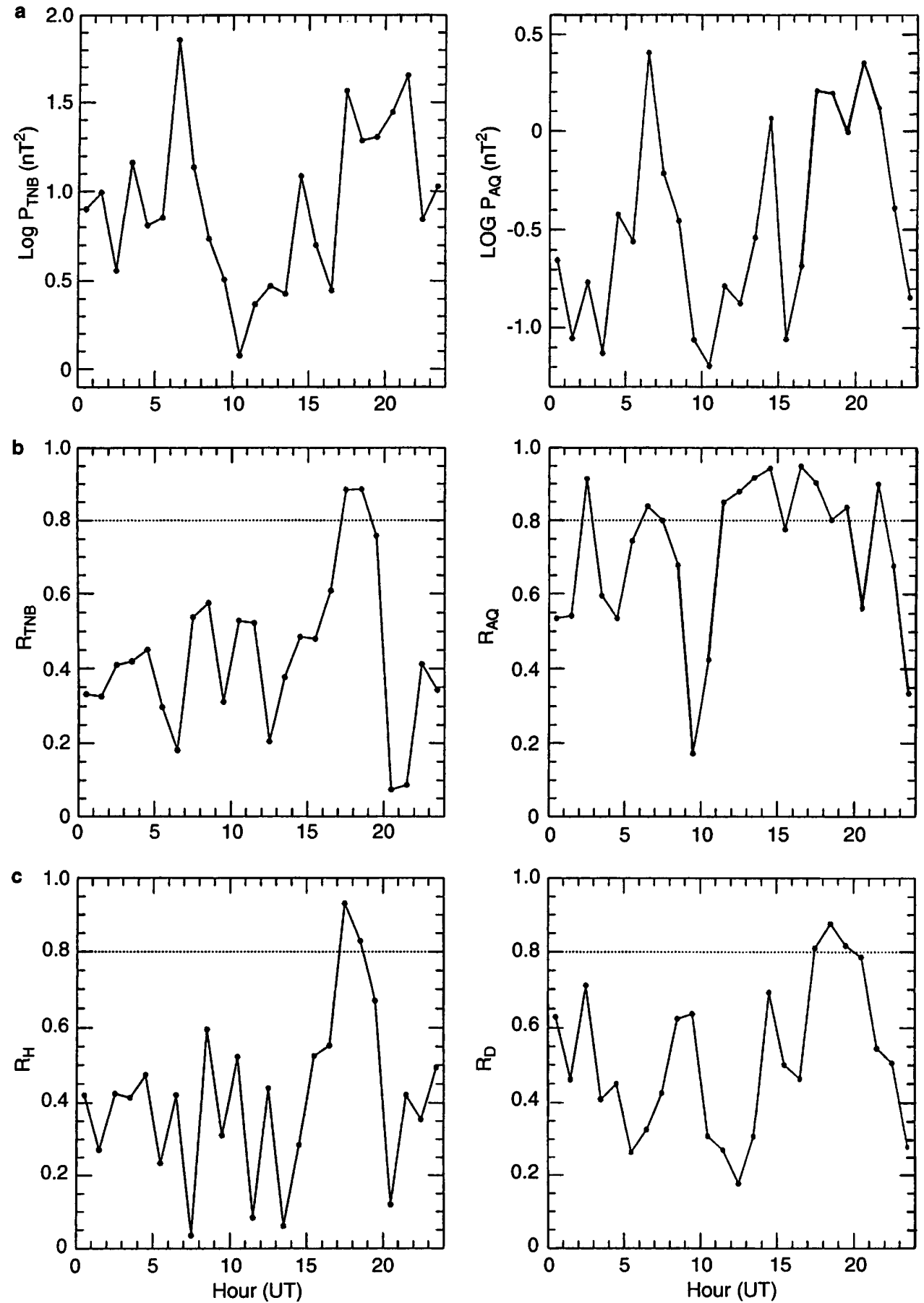
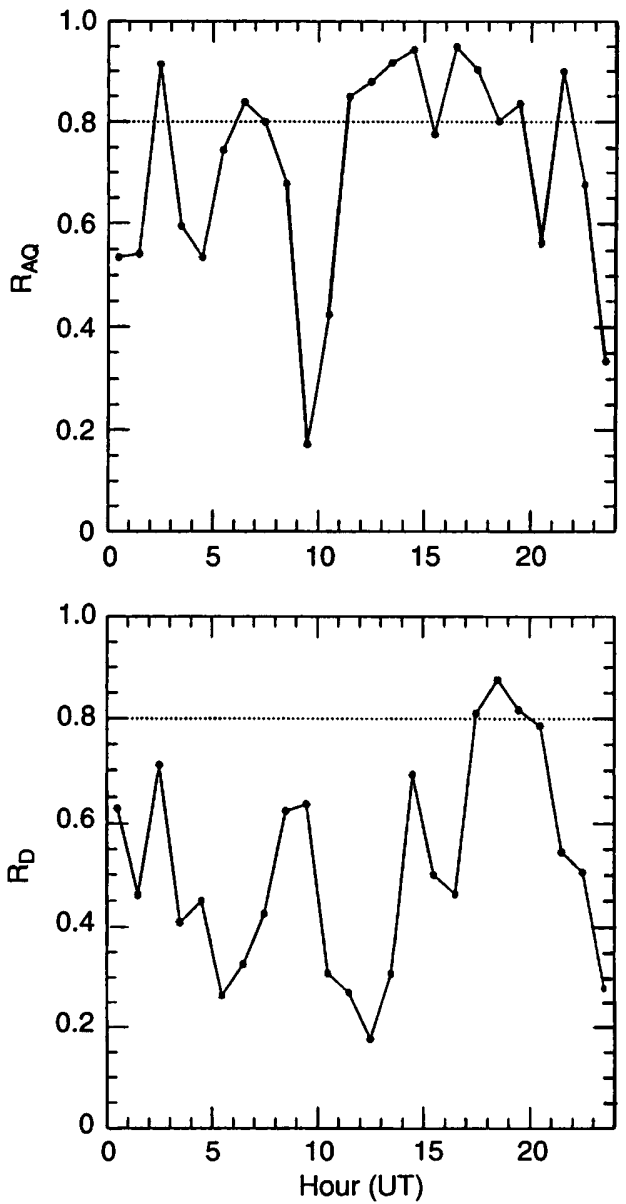

Fig. 2a-c. The pattern of hourly averaged a total power, $\mathbf{b}$ coherence between the horizontal components at each station and $\mathbf{c}$ coherence between the corresponding components at the two stations on April 11, 1997. All values are computed in the $1.9-5 \mathrm{mHz}$ frequency band 
the time behaviour of the hourly total $(\mathrm{H}+\mathrm{D})$ power in the $1.9-5 \mathrm{mHz}$ frequency band. As can be noted, although the two stations are at a very different magnetic local time, there is good correspondence between the power pattern at the two stations with simultaneous power enhancements at $\approx 06 \mathrm{UT}, \approx 14$ UT and $\approx 17-21 \mathrm{UT}$; in particular, the correlation coefficient between power levels over the whole day is 0.86 (well above a confidence level of $99.9 \%$ ). These results suggest that the fluctuation level is not a local phenomenon; it rather reflects global magnetospheric disturbances driven by the SW: indeed, all the observed power intensifications find correspondence in SW density enhancements detected from WIND about one hour before, as it can be seen from a comparison with Fig. 1.

An analysis of the signal coherence $\mathrm{R}$ between the two horizontal components in the $1.9-5 \mathrm{mHz}$ frequency band (Fig. 2b) shows that at AQ all the intervals with an high power level are typically characterized by an $\mathrm{R}$ value greater than 0.8 (the same technique applied to a random generated time series provides $\mathrm{R}>0.8$ only for less than $0.05 \%$ of cases). Conversely at TNB, far from the local geomagnetic noon (06-08 UT and 14-15 UT) and during southward IMF conditions (20-22 UT), the strong power intensifications do not correspond to coherent fluctuations; indeed the coherence reaches values higher than 0.8 only in the time interval $17-19$ UT, when the station is approaching the local geomagnetic noon $(\approx 20 \mathrm{UT})$ during northward IMF conditions. In a similar situation TNB, which is usually located in the polar cap, is expected to reach minimum distances from closed field lines; in this sense DMSP observations confirm that between 17 UT and 19 UT TNB was located just $\approx 2^{\circ}$ from the cusp. The coherence between the corresponding components at the two stations (Fig. 2c) sharply increases in the time interval 17-19 UT (i.e. when TNB is close to the cusp and AQ is in the evening sector) with peak values greater than 0.8 for both components, suggesting that pulsations with similar characteristics are simultaneously observed at AQ and TNB despite the wide latitudinal and longitudinal separation.

We conducted a more careful analysis of this time interval evaluating (by means of the maximum entropy method) the power spectra of the geomagnetic field components (Fig. 3a, b). As can be seen, major power peaks clearly emerge on both components and stations at the same frequencies, namely at $2.1-2.2,4.7-4.8 \mathrm{mHz}$ and, more particularly, at $3.6-3.7 \mathrm{mHz}$. In this sense it is interesting to remark that a comparison with simultaneous observations from magnetospheric spacecraft (GOES 8 and GOES 9, which are in geosynchronous orbits at local times UT-5 and UT-9, respectively) clearly reveals the occurrence of power peaks at approximately the same frequencies also in the magnetospheric field spectra (Fig. 3c).

A coherence analysis between the two stations for both components shows that the highest coherence $(\mathrm{R}>0.8)$ is associated with the $3.6 \mathrm{mHz}$ mode. Figure 4 shows the $3.6 \mathrm{mHz}$ filtered data observed on the ground and in the magnetosphere; as can be seen, all

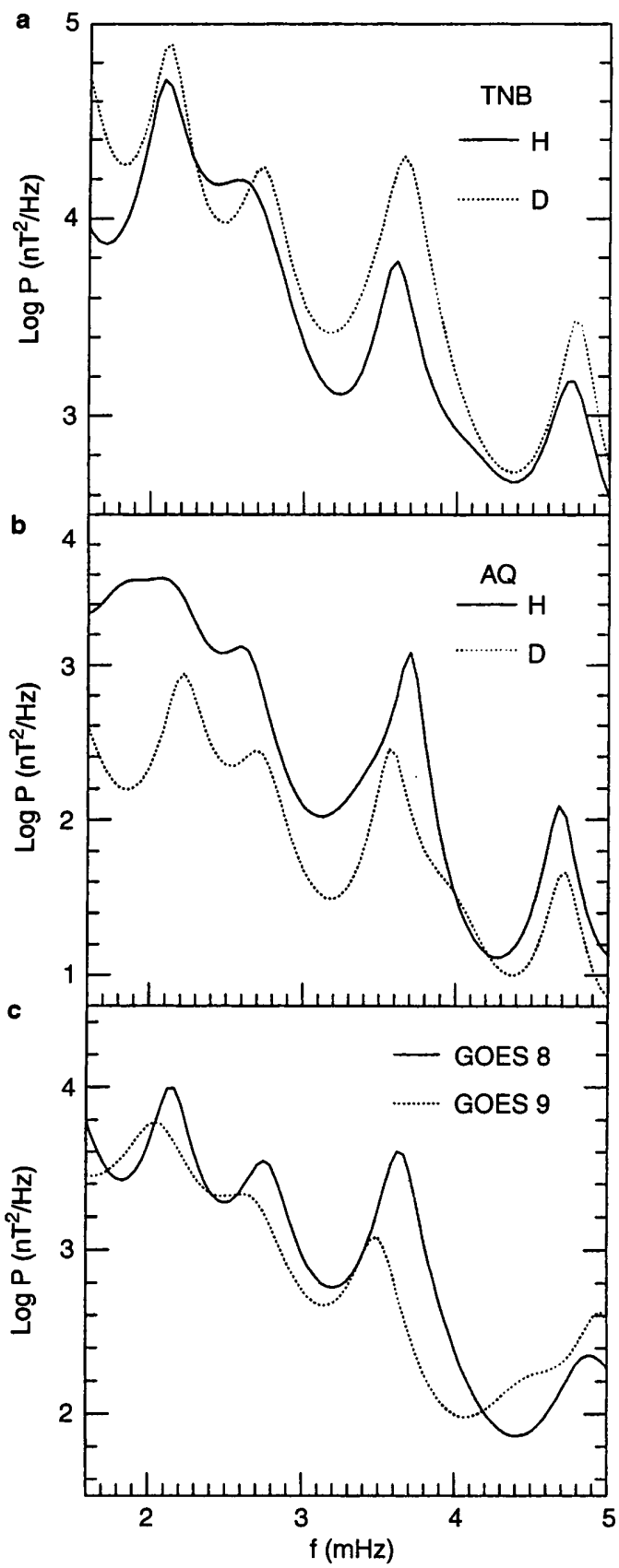

Fig. 3a-c. Power spectra of the horizontal components at a TNB, b AQ and $\mathbf{c}$ of the total magnetospheric field observed by GOES 8 and GOES 9 in the time interval 17-19 UT

observations show the simultaneous occurrence of a major wave packet starting at $\approx 1715$ UT and a smaller signal intensification at $\approx 1845$ UT.

We also analyzed the polarization sense of the ground $3.6 \mathrm{mHz}$ pulsations in the time interval $17-19$ UT (in Fig. 5 we show, as an example, the hodograms corresponding to the time interval 1730-1745 UT) and found that it is counterclockwise and clockwise at TNB and $\mathrm{AQ}$, respectively; moreover the observed orientations of the polarization ellipses at TNB and AQ are in the north-east and north-west direction, respectively. These results are consistent with the magnetic local time and latitude dependence of the polarization sense and 


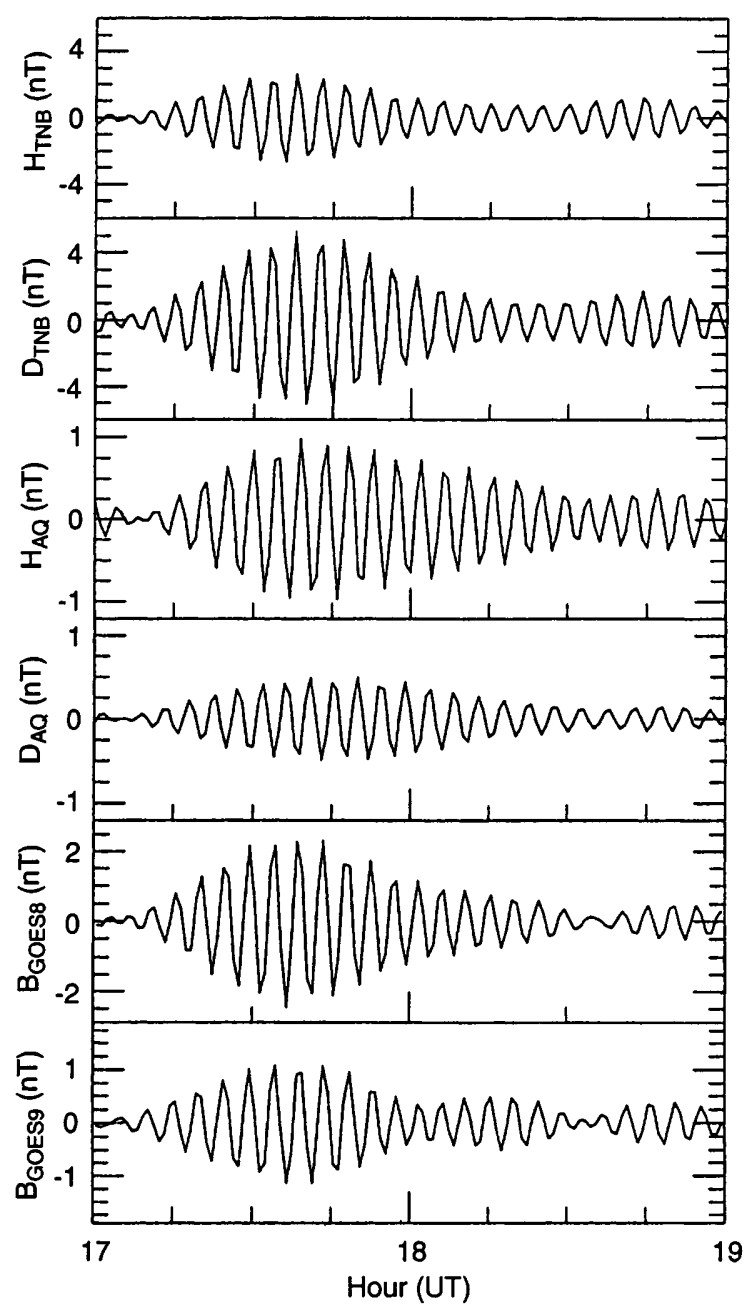

Fig. 4. The $3.6 \mathrm{mHz}$ filtered data at $\mathrm{TNB}$, AQ and in the magnetosphere in the time interval 17-19 UT

ellipse orientation of low frequency pulsations originally proposed by Samson (1972) as well as with the polarization pattern recently observed at TNB and AQ by Lepidi et al. (1998).

\section{Summary and discussion}

We analyzed the low frequency geomagnetic field fluctuations which were detected on April 11, 1997, a day characterized by the Earth's passage of a solar ejecta with northward IMF direction and variable SW pressure. We used data from a very high-latitude station, which is usually located in the polar cap, and a lowlatitude station, which is situated in the opposite hemisphere at a wide longitudinal separation.

The time behaviour of the fluctuation power at the two stations is very similar: it does not show any magnetic local time dependence and major SW pressure variations are followed at both stations by simultaneous low frequency power enhancements.

As is well known, polar cap stations are typically expected to reach minimum distances from closed field lines around local noon; moreover, during northward IMF conditions the polar cusp is expected to move poleward (Carbary and Meng, 1986). These conditions are the most favourable for polar cap stations to observe the same phenomena which are detected at low latitudes in presence of oscillation modes which extend to a major portion of the Earth's magnetosphere.

As matter of fact, we found that during major power enhancements, the coherence between the fluctuations at the two stations attains very high values during closed magnetospheric conditions and when TNB is close to the local geomagnetic noon; conversely, the coherence becomes low at a distance from the local geomagnetic noon at TNB and during open magnetospheric conditions. More particularly, when TNB was located approximately only $2^{\circ}$ poleward of the dayside cusp projection, the observed coherent fluctuations had common frequencies and simultaneous wave packets at ground stations as well as in the Earth's magnetosphere. In addition, the polarization characteristics were observed to be consistent with disturbances propagating westward in the local morning and eastward in the local afternoon (Southwood, 1974; Lepidi et al., 1998). The emerging overview would then suggest that these obser-
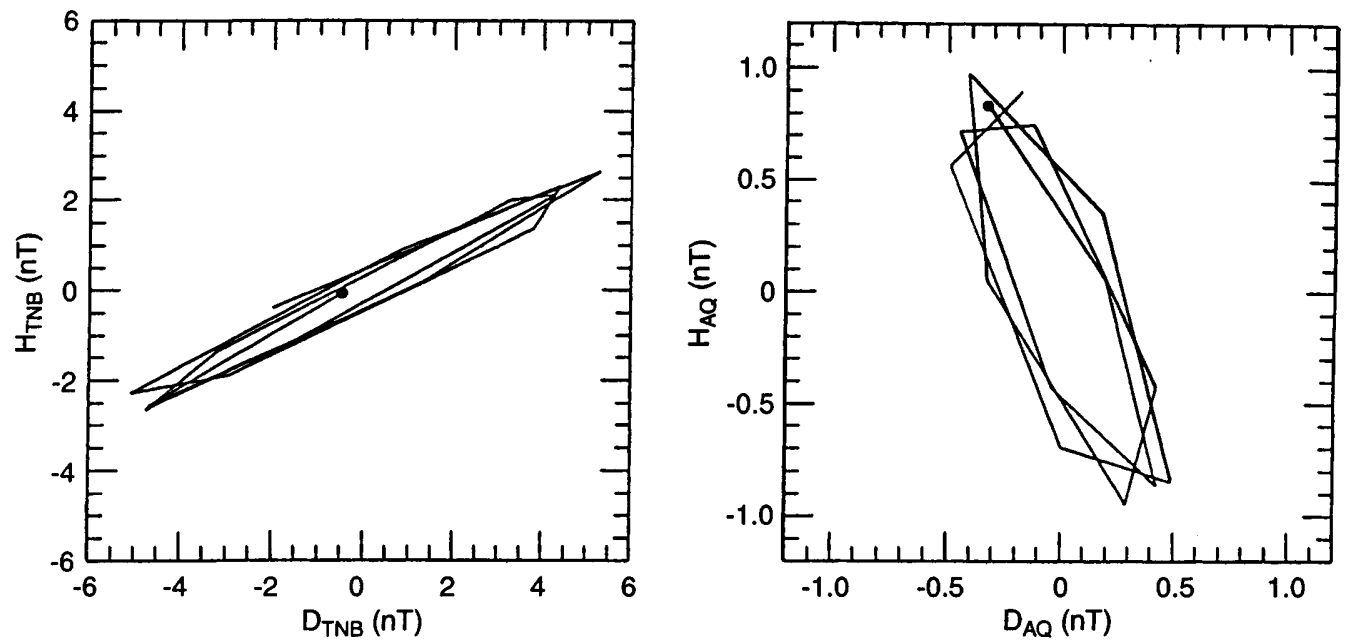

Fig. 5. Hodograms of the $3.6 \mathrm{mHz}$ mode at TNB (left panel) and AQ (right panel) in the time interval 1730-1745 UT; dots indicate the initial points 
vations can be interpreted in terms of compressional oscillations, driven by SW pressure pulses (Walker et al., 1992), which extend to a major portion of the Earth's magnetosphere. In this sense a comparison from a wide array of stations might be interesting for a careful analysis of similar global oscillations on a planetary scale. Evidence for similar long period oscillations has already been found in the analysis of a single event observed at the two stations during the passage of the January 10-11, 1997 magnetic cloud (Villante et al., 1998); moreover, previous statistical studies of geomagnetic ULF fluctuations at TNB (Villante et al., 1997) and AQ (Francia and Villante, 1997) have already shown evidence for stable power enhancements at discrete frequencies $(1.2-1.4,1.9,2.5-2.7$ and 3.3$3.6 \mathrm{mHz}$ ) which become more evident during daytime intervals: in this sense it is interesting that $3.6 \mathrm{mHz}$ is the frequency of the coherent oscillations we found in the present study.

Acknowledgements. This research activity at L'Aquila is supported by PNRA/CNR, GIFCO/CNR and MURST. The authors thank $H$. Singer at NOAA SEC and CDAWEB for providing GOES data. DMSP observations have been obtained from the corresponding website (scientist responsible: P. Newell).

Topical Editor R. Schwenn thanks D. G. Sibeck and C. J. Farrugia for their help in evaluating this paper.

\section{References}

Berdichevsky, D., J.-L. Bougeret, J.-P. Delaboudinière, N. Fox, M. Kaiser, R. Lepping, D. Michels, S. Plunkett, D. Reames, M. Reiner, I. Richardson, G. Rostoker, J. Steinberg, B. Thompson, and T. von Rosenvinge, Evidence for multiple ejecta; April 7-11, 1997 ISTP Sun-Earth connection event, Geophys. Res. Lett., 25, 2473, 1998.

Burlaga, L. F., Magnetic clouds, in Physics of the Inner Heliosphere, Eds. R. Schwenn and E. Marsch, vol 2, p. 1, SpringerVerlag, Berlin Heidelberg New York 1991.

Carbary, J. F., and C. I. Meng, Correlation of cusp latitude with Bz and AE (12) using nearly one year's data, J. Geophys. Res., 91, $10047,1986$.

Farrugia, C. J., M. P. Freeman, L. F. Burlaga, R. P. Lepping, and K. Takahashi, The Earth's response under continued forcing; substorm activity during the passage of an interplanetary magnetic cloud, J. Geophys. Res., 98, 7657, 1993.

Farrugia, C. J., R. P. Lepping, L. F. Burlaga, A. Szabo, D. Vassiliadis, P. Stauning, and M. P. Freeman, The WIND magnetic cloud of October 18-20, 1995: implications for the magnetosphere, EOS, Trans. Am. Geophys. Un., 77 (17), S241, 1996.

Francia, P., and U. Villante, Some evidence of ground power enhancements at frequencies of global magnetospheric modes at low latitude, Ann. Geophysicae, 15, 17, 1997.

Francia, P., S. Lepidi, U. Villante, and A. J. Lazarus, Geomagnetic response at low latitude to multiple solar wind pressure variations, in press. J. Geophys. Res., 1999.

Gosling, J. T., Coronal mass ejections and magnetic flux ropes in interplanetary space, in Physics of Magnetic Flux Ropes, Geophys. Monogr. Ser., vol 58, Eds. C.T. Russell et al., 343, AGU, Washington, D.C., 1990.

Lepidi, S., P. Francia, U. Villante, L. J. Lanzerotti, and A. Meloni, Polarization pattern of low frequency geomagnetic field fluctuations $(0.8-3.6 \mathrm{mHz})$ at high and low latitude, J. Geophys. Res., 104, 305, 1999.

Lepping, R. P., L. F. Burlaga, B. T. Tsurutani, K. W. Ogilvie, A. J. Lazarus, D. S. Evans, and L. W. Klein, The interaction of a very large interplanetary magnetic cloud with the magnetosphere and with cosmic rays, J. Geophys. Res., 96, $9425,1991$.

Meloni, A., and F. Molina, Study of the S variation at L'Aquila Geomagnetic observatory, Ann. Geophysicae, 7, 387, 1989.

Russell, C. T., and M. Ginskey, Sudden impulses at subauroral latitudes: response for northward interplanetary magnetic field, J. Geophys. Res., 100, 23 695, 1995.

Russell, C. T., M. Ginskey, and S. M. Petrinec, Sudden impulses at low latitude stations: steady state response for northward interplanetary magnetic field, J. Geophys. Res., 99, 253, 1994.

Samson, J. C., Three-dimensional polarization characteristics of high-latitude Pc5 geomagnetic micropulsations, J. Geophys. Res., 77, 6145, 1972.

Sibeck, D. G., W. Baumjohann, R. C. Elphic, D. H. Fairfield, J. F. Fennell, W. B. Gail, L. J. Lanzerotti, R. E. Lopez, H. Luehr, A. T. Y. Lui, C. G. Maclennan, R. W. McEntire, T. A. Potemra, T. J. Rosenberg, and K. Takahashi, The magnetospheric response to 8-minute period strong amplitude upstream pressure variations, J. Geophys. Res., 94, 2505, 1989.

Southwood, D. J., Some features of field line resonances in the magnetosphere, Planet. Space Sci., 22, 483, 1974.

Villante, U., S. Lepidi, P. Francia, P. Palangio, and A. Meloni, Long period geomagnetic field fluctuations at Terra Nova Bay (Antarctica), Geophys. Res. Lett., 24, 1443, 1997.

Villante, U., P. Francia, S. Lepidi, M. De Lauretis, E. Pietropaolo, L. Cafarella, A. Meloni, A. J. Lazarus, R. P. Lepping, and F. Mariani, Geomagnetic field variations at low and high latitude during the January 10-11, 1997 magnetic cloud, Geophys. Res. Lett., 25, 2593, 1998.

Walker A. D. M., J. M. Ruohoniemi, K. B. Baker, R. A. Greenwald and J. C. Samson, Spatial and temporal behavior of ULF pulsations observed by the Goose Bay HF radar, J. Geophys. Res., 97, 12 187, 1992. 